\title{
Erratum to: Romidepsin for the treatment of relapsed/refractory peripheral T cell lymphoma: prolonged stable disease provides clinical benefits for patients in the pivotal trial
}

Francine Foss ${ }^{1 *}$, Steven Horwitz ${ }^{2}$, Barbara Pro ${ }^{3}$, H. Miles Prince ${ }^{4}$, Lubomir Sokol ${ }^{5}$, Barbara Balser ${ }^{6}$, Julie Wolfson ${ }^{6}$ and Bertrand Coiffier ${ }^{7}$

\section{Erratum}

The original article [1] contains an error whereby Fig. 2a $\& \mathrm{~b}$ are mistakenly interchanged and contain incorrect colour-coding.

The correct versions of Fig. 2a \& b are thus displayed below.

\footnotetext{
Author details

${ }^{1}$ Yale Cancer Center, 333 Cedar St, TMP 3, PO Box 208028, New Haven, CT 06520-8028, USA. ${ }^{2}$ Memorial Sloan-Kettering Cancer Center, New York, NY, USA. ${ }^{3}$ Kimmel Cancer Center, Thomas Jefferson University, Philadelphia, PA, USA. ${ }^{4}$ Peter MacCallum Cancer Centre, University of Melbourne, Melbourne, Australia. ${ }^{5}$ Moffitt Cancer Center, Tampa, FL, USA. ${ }^{6}$ Veristat, LLC, Southborough, MA, USA. ${ }^{7}$ Hospices Civils de Lyon, Lyon, France.
}

Received: 21 July 2017 Accepted: 14 August 2017 Published online: 18 September 2017

\section{Reference}

1. Foss F, et al. Romidepsin for the treatment of relapsed/refractory peripheral

T cell lymphoma: prolonged stable disease provides clinical benefits for patients in the pivotal trial. J Hematol Oncol. 2016;9:22.

\footnotetext{
* Correspondence: francine.foss@yale.edu

${ }^{1}$ Yale Cancer Center, 333 Cedar St, TMP 3, PO Box 208028, New Haven, CT 06520-8028, USA

Full list of author information is available at the end of the article
} 


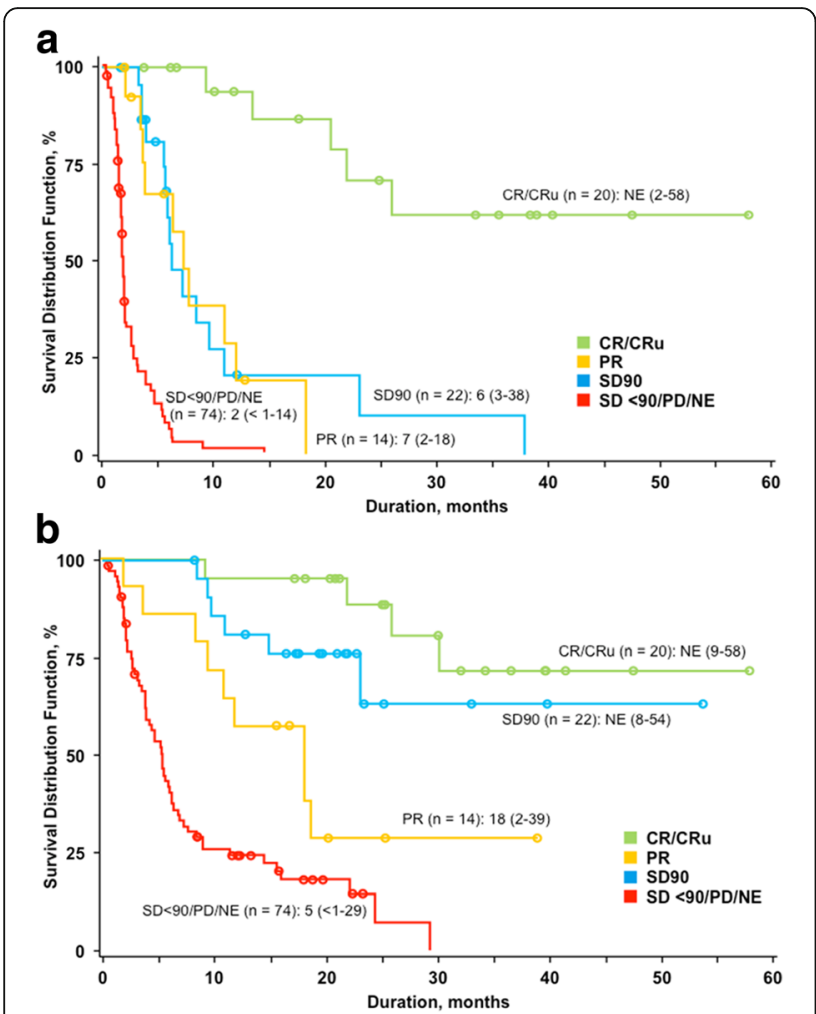

Fig. 2: Survival based on clinical IRC assessment by best response to romidepsin $(n=130)$. Progression-free survival (a) and overall survival (b). Patients with insufficient efficacy data to determine response due to early termination ( $N E ; n=29$ ) were included as nonresponders. NE not evaluable 